\title{
Strange quark matter: mapping QCD lattice results to finite baryon density by a quasi-particle model
}

\author{
B Kämpfer*, A Peshier狎 and G Soff§ \\ * Forschungszentrum Rossendorf, D-01314 Dresden, PF 510119, Germany \\ If Dept. of Physics, Brookhaven Nat. Lab., Upton, NY 11973, USA \\ $\S$ Institut für Theoretische Physik, TU Dresden, D-01062 Dresden, Germany
}

\begin{abstract}
A quasi-particle model is presented which describes QCD lattice results for the 0,2 and 4 quark-flavor equation of state. The results are mapped to finite baryo-chemical potentials. As an application of the model we make a prediction of deconfined matter with appropriate inclusion of strange quarks and consider pure quark stars.
\end{abstract}

\section{Introduction}

The QCD lattice calculations of the equation of state (EoS) of deconfined matter have advanced to such a level that reliable results for the pure gluon plasma are available [1]. For the two-flavor case an estimate of the continuum extrapolation is at disposal [2]. The EoS of four light flavors [3] are not yet continuum extrapolated. The physically interesting case of two light flavors and a medium-heavy strange quark is still in progress [4]. All of these ab initio calculations of the EoS of deconfined matter, however, are yet constrained to finite temperatures $T \geq T_{c}$ (here $T_{c}$ is the deconfinement temperature) and vanishing baryo-chemical potential, $\mu=0$. While first attempts to calculate the $\operatorname{EoS}$ at $\mu \neq 0$ are under way [5], the final results can probably be expected only in a few years. It is therefore a challenge to attempt an extrapolation of the QCD lattice results into the domain of finite baryo-chemical potential. Here we employ a quasi-particle model to accomplish this goal.

Quasi-particle models have proven powerful in describing properties of strongly correlated systems in condensed matter physics. Analogously, one should expect that also strongly interacting matter can be described effectively within quasi-particle models. Indeed, the investigations of $\phi$-derivable self-consistent approximations [6], combined with hard thermal loop resummation [7], delivered recently compelling support of a quasi-particle description of deconfined matter. Starting from the QCD Lagrangian a chain of approximations is derived [8] which results in a quasi-particle model of deconfined matter agreeing with lattice results at $T>2.5 T_{c}$. On the other hand, employing the hard thermal/dense loop resummation at finite baryo-chemical potential, further evidence for a quasi-particle description of cold deconfined matter is gained [9]. What is still needed is an interpolating model, which reproduces the QCD lattice data down to $T_{c}$ and, at the same time, extrapolates to finite values of $\mu$ even up to $T=0$. We present here such a model and apply it to calculate static properties of cold, pure quark stars with strange quarks properly taken into account.

$\dagger$ Supported by BMBF 06DR921 and GSI.

+ Supported by A. v. Humboldt foundation. 


\section{Quasi-particle model of deconfined matter}

With increasing sophistication of QCD lattice calculations of the EoS also phenomenological quasi-particle models have been developed [10. Of central importance to our model [11] are the baryon density $n$ and the entropy density $s$ as quantities which are dominated by the quasi-particle structure of the interacting system. . Approximating the self-energies of the quarks $(q)$, with a current mass $m_{q 0}$, and the gluons $(g)$ by the gauge-invariant asymptotic values of the 1-loop expressions

$$
\begin{aligned}
& \Pi_{q}^{*}=2 \omega_{q}\left(m_{q 0}+\omega_{q}\right), \quad \omega_{q}^{2}=\frac{1}{6}\left[T^{2}+\frac{\mu_{q}^{2}}{\pi^{2}}\right] g_{\mathrm{eff}}^{2}, \\
& \Pi_{g}^{*}=\frac{1}{6}\left[\left(3+\frac{1}{2} N_{f}\right) T^{2}+\frac{3}{2 \pi^{2}} \sum_{q} \mu_{q}^{2}\right] g_{\mathrm{eff}}^{2},
\end{aligned}
$$

the densities are given by the standard formulae of ideal gases (labeled by the superscript "id") of quarks and gluons with effective masses $m_{i}^{2}=m_{i 0}^{2}+\Pi_{i}^{*}, i=(q, g)$,

$$
\begin{aligned}
& n=\sum_{q}\left\{n_{q}^{\mathrm{id}}\left(T, \mu ; m_{q}[T, \mu]\right)-n_{\bar{q}}^{\mathrm{id}}\left(T, \mu ; m_{q}[T, \mu]\right)\right\}, \\
& s=s_{g}^{\mathrm{id}}\left(T, \mu ; m_{g}[T, \mu]\right)+\sum_{q} s_{q}^{\mathrm{id}}\left(T, \mu ; m_{q}[T, \mu]\right) .
\end{aligned}
$$

Beyond this resummation of the leading-order contributions, non-perturbative effects are described in the phenomenological quasi-particle model by the effective coupling $g_{\text {eff }}$. The requirement $g_{\text {eff }}(T, \mu) \rightarrow g_{1-\text { loop }}$ at large values of $T$ and/or $\mu$ ensures the smooth transition to the asymptotic regime.

The corresponding pressure $p$ and energy density $e$ are $p=\sum_{i} p_{i}^{\text {id }}\left(T, \mu ; m_{i}[T, \mu]\right)-$ $B(T, \mu)$ and $e=\sum_{i} e_{i}^{\text {id }}\left(T, \mu ; m_{i}[T, \mu]\right)+B(T, \mu)$. The quantity $B(T, \mu)$ is not an independent quantity but obtained by integrating $\left(\partial p^{\text {id }} / \partial m_{i}^{2}\right)\left(\partial \Pi_{i}^{*} / \partial T\right)=(\partial B / \partial T)$, $\left(\partial p^{\text {id }} / \partial m_{i}^{2}\right)\left(\partial \Pi_{i}^{*} / \partial \mu\right)=(\partial B / \partial \mu)$ which come from the stationarity condition $\delta p / \delta \Pi_{i}=0[12]$.

Let us mention two implications of the quasi-particle model. (i) According to the Feynman-Hellmann relation the chiral condensate is given by $\left\langle\bar{\Psi}_{q} \Psi_{q}\right\rangle \propto \partial p / \partial m_{q 0} \rightarrow$ 0 for $m_{q 0} \rightarrow 0$, i.e. for vanishing current quark masses the chiral condensate vanishes in agreement with the chiral symmetry restoration at $T>T_{c}$. (ii) In the asymptotic region, $T \rightarrow \infty$ and $\mu \rightarrow \infty$, an expansion in the coupling yields $p(T, \mu)=p_{0}(T, \mu)+p_{2}(T, \mu)+\cdots$ thus reproducing the perturbative results 13 in the orders of $g^{0}$ and $g^{2}$.

\section{Tests of the model}

For the effective coupling strength $g_{\text {eff }}$ we chose a regularized parameterization of the 1-loop running coupling strength. The resulting comparison with the QCD lattice data is displayed in figure 1 for various flavor numbers $N_{f}$. Notice the almost perfect agreement with the data which ensures that the other thermodynamical quantities are also nicely reproduced (for details cf. [1]).

* This is supported by the $\phi$-derivable approach 6 where corrections to the quasi-particle picture arise only beyond the resummed leading-loop order contributions [8, 14]. Within the massless $\varphi^{4}$ theory the form of $s$ below is obtained by employing the Luttinger-Ward theorem [15] with a superdaisy resummed propagator and a double-ring $\phi$ functional 16$]$. 

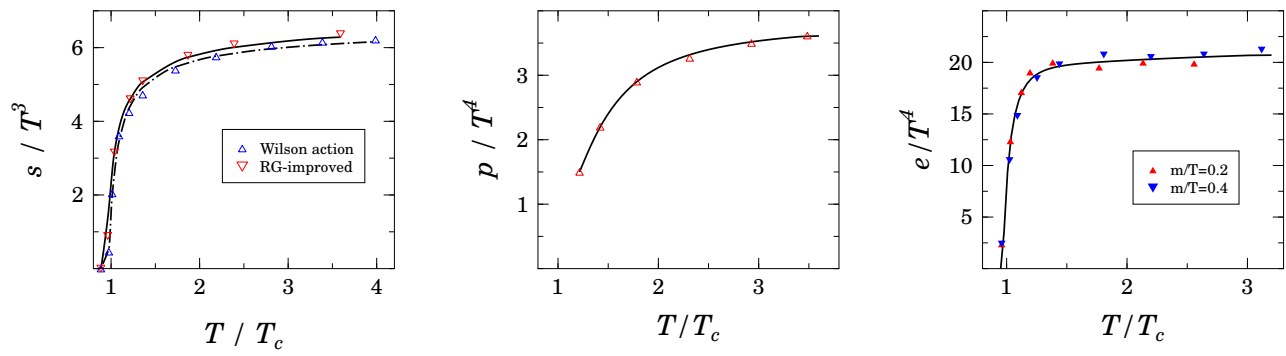

Figure 1. Comparison of the quasi-particle model with QCD lattice results for

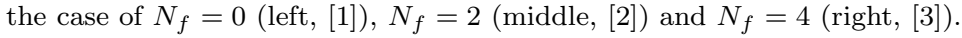

\section{Extrapolation to $\mu>0$}

Our model assumes the validity of the underlying quasi-particle structure at finite $\mu$. Some limitation of this assumption may arise from the recently debated suprafluidity and color-flavor locking effects at small temperatures and asymptotically large chemical potentials [17]. However, since many of the discussed effects [17], which also depend sensitively on the actual strange quark mass (as the phase diagram does already at $\mu=0$ [18]), are near the Fermi surface, the gross properties of the EoS might not be modified strongly.

Since the pressure $p$ is a potential it has to fulfill the Maxwell relation $\partial n / \partial T=$ $\partial s / \partial \mu$, which results in a partial differential equation for the coupling of the form

$$
a_{T} \frac{\partial g_{\mathrm{eff}}^{2}}{\partial T}+a_{\mu} \frac{\partial g_{\mathrm{eff}}^{2}}{\partial \mu}=b .
$$

A solution for the 4-flavor lattice case [3] is displayed in the left panel of figure 2. The strong increase of $g_{\text {eff }}$ towards smaller values of $T$ and $\mu$ can be considered as an indication of approaching the phase boundary. Despite of the fact that our model does not allow to make statements on the nature of the deconfinement transition, one can safely conclude that the region of smaller values of $T$ and $\mu$, not covered by the elliptic characteristics shown in the right panel of figure 2, may be related to confined matter.
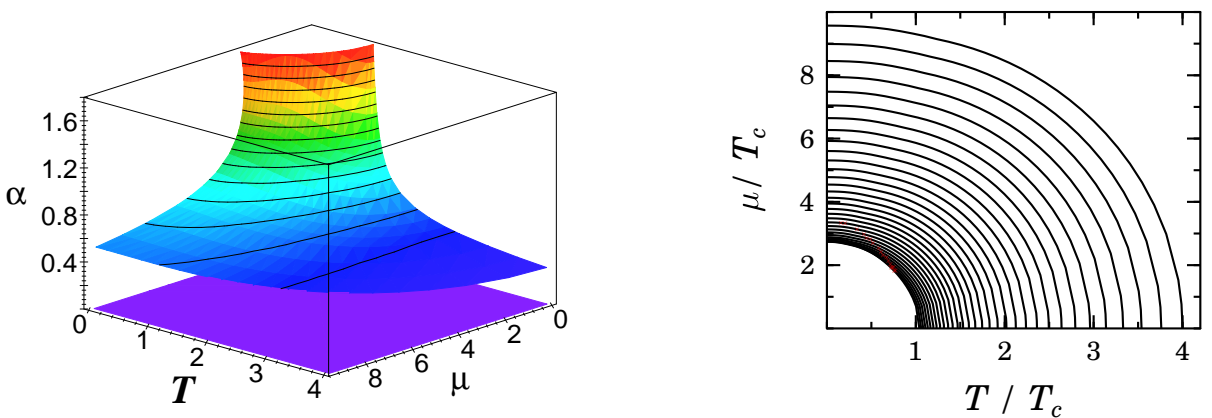

Figure 2. Left panel: The effective coupling strength $\alpha=g_{\text {eff }}^{2} /(4 \pi)$ as a function of $T$ and $\mu$ (both in units of $T_{c}$ ) Right panel: The characteristics in solving equation (5). 
The order of magnitude of the baryo-chemical potential $\mu_{B}=3 \mu$ for the innermost characteristics at $T=0$ is $1.4 \mathrm{GeV}$ when choosing the scale $T_{c}=160 \mathrm{MeV}$.

Thermodynamic state variables are available in tabular form in [19]. At small $T$, the pressure in the small- $\mu$ region becomes negative giving a lower bound for the existence of conventional deconfined matter. The energy per baryon, however, at the point of vanishing pressure is quite large, typically in the order of $1.5 \mathrm{GeV}$ for $N_{f}=2,3,4$. That means, even when including strangeness, no hint to stable strangelets arises from our model.

\section{Pure quark stars with strangeness}

To condense the properties of the predicted $\mathrm{EoS}$ in a few concise numbers let us consider pure quark stars with locally charge-neutral deconfined matter in $\beta$ equilibrium. The chemical potentials of $\mathrm{u}, \mathrm{d}, \mathrm{s}$ quarks and leptons $(l)$ are related according to $\mu_{s}=\mu_{d}=\mu_{u}+\mu_{l} \equiv \mu$. Unfortunately, continuum extrapolated QCD lattice data for the $2+1$ flavor case with physical quark masses are not yet at disposal at $T \neq 0$. Therefore, we first guess sets of EoS for the $2+1$ flavor case at $\mu=0$ and then extrapolate them to finite $\mu$ at $T=0$. In doing so we are guided by the parameter range obtained in 11]. Adding to the pressure the lepton contribution it turns out that the energy density - pressure relation obtained numerically can be approximated by $e=4 \tilde{B}+\tilde{\alpha} p$ with $\tilde{\alpha}=3.1 \cdots 4.5$ and $\tilde{B}^{1 / 4} \geq 200 \mathrm{MeV}$. Note that $\tilde{B}^{1 / 4}$ is not longer an ad hoc parameter in the spirit of the bag model but a parameter which will be fixed by improving the QCD lattice data for the $2+1$ flavor case [4].

Integrating the Tolman-Oppenheimer-Volkoff equations for spherical stars in hydrodynamical equilibrium up to vanishing pressure at the surface one gets the masses and radii as displayed in figure 3. Since the estimated range of $\tilde{B}^{1 / 4}$ is $>200 \mathrm{MeV}$, the strange quark stars have small masses $\left(M \leq 1 M_{\odot}\right)$ and radii $(R \leq 7 \mathrm{~km})$.

It should be emphasized that our model does not predict absolutely stable (strange) quark matter. Rather, at smaller values of $\mu$ and $p>0$ the transition to hadron matter is expected to happen. The static properties of the corresponding hybrid stars depend sensitively on the nature of the confinement transition and details of the hadronic EoS.

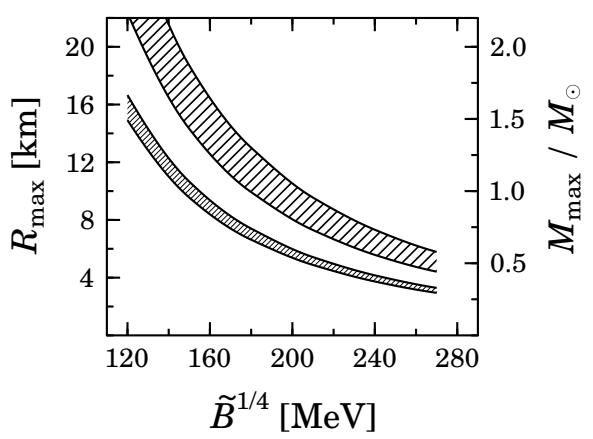

Figure 3. Maximum masses (upper hatched band, right scale) and corresponding radii (lower hatched band, left scale) of pure quark stars as a function of $\tilde{B}^{1 / 4}$. The upper (lower) limit of the bands is for $\tilde{\alpha}=3(4.5)$. 


\section{Summary}

In summary we present a quasi-particle model for the EoS of purely deconfined matter, which describes the available QCD lattice calculations at $\mu=0$ and $T \geq T_{c}$, and map this to finite values of the baryo-chemical potential including properly the strangeness degree of freedom. The model does not point to stable strangelets. The resulting cold, pure quark stars with strangeness are light and small.

At finite temperatures the strange quarks do not play any exceptional role: the self-energies mask nearly all of the finite-mass effect, even having used here $m_{s 0}=150$ $\mathrm{MeV}$, while recent lattice calculations point to smaller values [20]. This is in agreement with QCD lattice calculations which display for $p / p_{0}$ almost the same values for the 2,3 and $2+1$ flavor cases $\left[1\right.$. The gluon abundance near $T_{c}$ is strongly suppressed.

\section{References}

[1] Boyd G, Engels J, Karsch F, Laermann E, Legeland C, Lütgemeier M and Petersson B 1996 Nucl. Phys. B 469419

Okamoto M et al (CP-PACS collaboration) 1999 Phys. Rev. D 60094510

[2] Karsch F 2000 Nucl. Phys. Proc. Suppl. 83-84 14

[3] Engels J, Joswig R, Karsch, Laermann E, Lütgemeier M and Petersson B 1997 Phys. Lett. B 396210

[4] Karsch F, Laermann E and Peikert A 2000 Phys. Lett. B 478447

[5] Engels J, Kaczmarek O, Karsch F and Laermann E 1999 Nucl. Phys. B 558307

[6] Baym G 1962 Phys. Rev. 1271391

[7] Andersen J O, Braaten E and Strickland M 2000 Phys. Rev. D 61 074016, Phys. Rev. D 61 014017, 1999 Phys. Rev. Lett. 832139

[8] Blaizot J P, Iancy E and Rebhan A 1999 Phys. Lett. B 470 181, Phys. Rev. Lett. 83 2906, hep-ph/0005003

[9] Baier R and Redlich K 2000 Phys. Rev. Lett. 842100

[10] Biro T S, Levai P and Müller B 1990 Phys. Rev. D 423078 Goloviznin V and Satz H 1993 Z. Phys. C 57671

Peshier A, Kämpfer B, Pavlenko O P and Soff G 1994 Phys. Lett. B 337235

Peshier A, Kämpfer B, Pavlenko O P and Soff G 1996 Phys. Rev. D 542399

Schertler K, Greiner C and Thoma M H 1997 Nucl. Phys. A 616659

Levai P and Heinz U 1998 Phys. Rev. C 571879

[11] Peshier A, Kämpfer B and Soff G 2000 Phys. Rev. C 61045203

[12] Lee T D and Yang C N 1960 Phys. Rev. 11722

[13] Freedman B A and McLerran L D 1977 Phys. Rev. D 16 1130, 1147, 1169 Kapusta J I 1989 Finite-Temperature Field Theory Cambridge University Press Zhai C and Kastening B 1995 Phys. Rev. D 527232

[14] Riedel E 1968 Z. Phys. 210403 Vanderheyden B and Baym G 1998 J. Stat. Phys. 93843

[15] Luttinger J M and Ward J C 1960 Phys. Rev. 1181417

[16] Peshier A, Kämpfer B, Soff G and Pavlenko O P 1998 Europhys. Lett. 43381

[17] Rajagopal K 1999 Nucl. Phys. A 661 150c Alford M, Rajagopal K and Wilczek F 1999 Nucl. Phys. B 537443 Berges J and Rajagopal K 1999 Nucl. Phys. B 538215

[18] Peikert A, Karsch F, Laermann E and Sturm B 1999 Nucl. Phys. Proc. Suppl. 73468

[19] www.fz-rossendorf.de/FWK/MITARB/Peshier/EoS.html

[20] Garden J et al (ALPHA and UKQCD Collaboration) 2000 Nucl. Phys. B 571237 